\title{
A FAST GLOBAL BEAM POSITION FEEDBACK SYSTEM FOR SUPER-ACO*
}

\author{
L. Cassinari", J. Darpentigny, J.-N. Maymon, A. Nadji, D. Pédeau \\ LURE, Université Paris-Sud, Bât. 209A, BP 34, 91898 Orsay Cedex, France
}

\section{Abstract}

A fast global feedback system has been installed on Super-ACO in order to improve beam stability. The feedback uses all the 16 BPMs of the machine and 8 correctors in each plane. Two different correction algorithms have been tested, an harmonic based method and a direct inversion of the response matrix using SVD technique. The fast corrections are computed by 8 DSPs connected by a fast digital data link in order to achieve a correction bandwidth exceeding $100 \mathrm{~Hz}$. Each DSP acquires the data for both planes of 2 BPMs and computes the output correction for 2 correctors. In this paper, we present the current status of the implementation of the system. We give the results on the efficiency of the orbit correction and the improvements on the photon beam stability.

\section{INTRODUCTION}

Among other recent developments on the Super-ACO storage ring, improvement in the orbit stability constitutes a major goal. The starting point of this work was the characterization of the beam position instability [1]. The orbit drift during operational runs has been measured several times at each BPM, and analysed. The spectrum analysis of the beam motion has also been measured at each BPM, and on a photon beam line, revealing several peaks in the horizontal plane, about from 10 to $30 \mathrm{~Hz}$.

In this paper, we will present the analysis of the beam motion, the implementation and the operation of the global feedback system, as well as the home developped acquisition and computation boards, based on DSP.

\section{SYSTEM OVERVIEW}

The system uses all the 16 BPMs of the machine, and 8 correctors in each plane to correct the orbit at a $16 \mathrm{kHz}$ rate, in order to achieve the correction bandwidth from DC to $100 \mathrm{~Hz}$, both in horizontal and vertical plane. Figure 1 shows the layout of the global feedback system.

The BPMs consist of four-button monitors placed between the quadrupoles of each doublet, at each side of the 8 straight sections.

* Work supported by CNRS, CEA and MENESR

\# Email : cassinari@lure.u-psud.fr

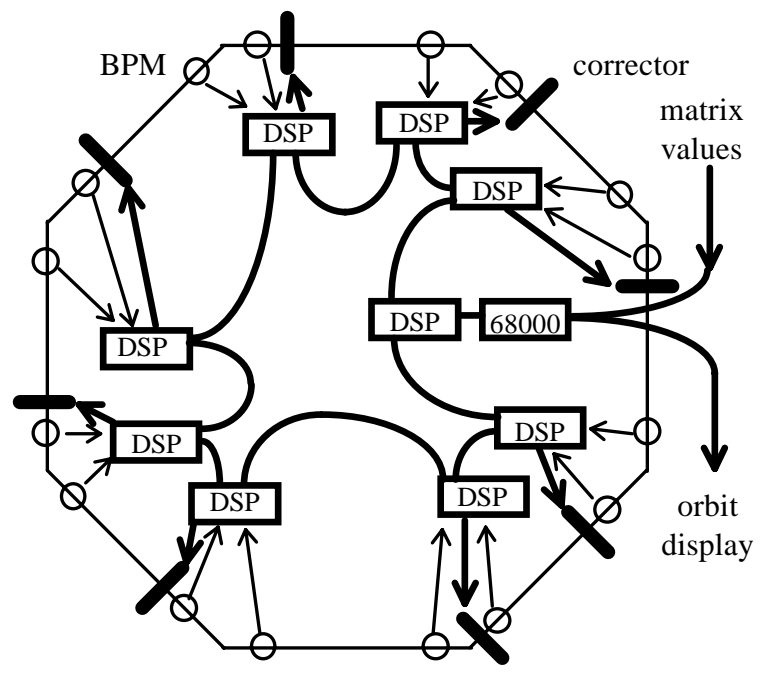

Fig. 1. Layout of the global feedback system.

\subsection{Beam Position Measurement}

The peak detector originally installed at the beginning of Super-ACO has been replaced 2 years ago by 16 BPM signal processors (one for each BPM) developped by BERGOZ Precision Beam Instrumentation. They consist of a four channel multiplexer, a heterodyne receiver with frequency synthetiser of the local oscillator, phase locked synchronous detector and low noise preamplifier with automatic gain control [2]. For Super-ACO, the processors use the 2nd RF harmonic of the picked-up signals (i.e. $199.756 \mathrm{MHz}$ ). The sampling frequency has been set to $33 \mathrm{kHz}$ by external clock, in order to avoid any aliasing problem related with synchrotron oscillations.

Each processor delivers $\mathrm{X}$ and $\mathrm{Z}$ quasi-continuous output signals which allow to analyse the beam motion noise up to several hundreds of $\mathrm{Hz}$. The achieved resolution is of $0.5 \mu \mathrm{m} / \mathrm{JHz}$ over the full operating range, from 0.5 to $500 \mathrm{~mA}$.

\subsection{Digital Signal Processing}

The digital processing system is home developped. Eight DSP boards acquire the $\mathrm{X}$ and $\mathrm{Z}$ signals from the 16 BPM processors (2 stations per DSP). They include two 16 bit MAX195 ADC, a four-channel MAX536 DAC, HDMP-1014/1012 low cost, high speed serial transmiter/receiver ; the DSP is an ADSP2 115. 
The 8 DSP boards are linked via fast high rate serial links and form a ring controlled by a DSP master board.

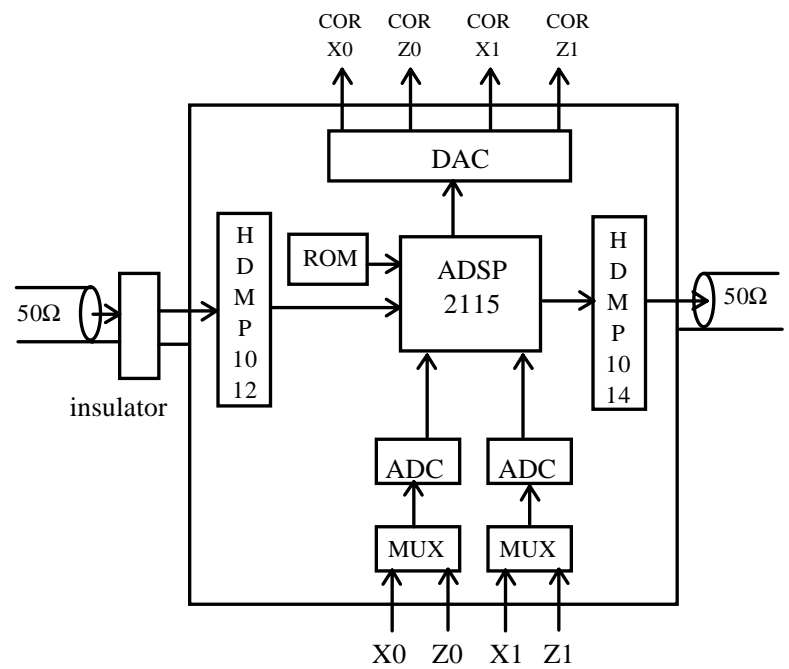

Fig. 2. DSP board.

The master controls the transmission cycle and ensures external communications. It delivers the orbit measurement to the general operating system. Its messages to the ring include the reference orbit, the coefficients of the matrix used for correction, and the status of the feedback (on/off, valid/non-valid).

During each cycle, each DSP board gets all the updated orbit data all along the ring, in order to calculate the correction needed for each corrector.

Each DSP board gets back the data it has delivered, and checks the conformity with the message sent ; in case of problem, the cycle is ignored (no change in the output). It takes $60 \mu$ s to complete the whole acquisition, transmission, checking and calculation cycle for the 32 data (16 X and $16 \mathrm{Z}$ ). Data concerning the reference orbit and matrix coefficient are permanently transmitted by the master, at a rate of 4 values per cycle (it takes 136 cycles to ensure the full updating of all the data).

\subsection{Orbit Corrections}

The correction kicks are produced by 8 coils in each transverse plane. These coils are mounted in the quadrupole magnets, in addition to the dipolar correctors used to correct the orbit at the beginning of every run. They are powered by current-controlled wideband power amplifiers.

\section{BEAM TESTS}

\subsection{Beam Behaviour without Feedback}

The orbit stability is affected by 2 main parameters : long term drifts and low frequency fluctuations.

In Super-ACO, drifts occuring with usual beam intensity decay can be as large as $500 \mu \mathrm{m}$ in the horizontal plane and $100 \mu \mathrm{m}$ in the vertical plane (with respective rms values of 300 and $50 \mu \mathrm{m}$ ) [3].

In one case, it has been possible to show that thermal distortion of the vacuum chamber pushing the quadrupole magnet, was responsible of a large amount of the horizontal drifts [4]. This problem has been cured, yielding to a reduction of drift excursions, but most of the origins of these drifts are still unknown.

Currently, an automatic correction in the horizontal plane is applied every $3 \mathrm{~min}$, in order to keep orbit drifts inside a max tolerance of $100 \mu \mathrm{m}$.

Noise spectrum of position measurement shows a different look in horizontal and vertical plane (see Fig. 3). While in the vertical plane, the only noticeable noise line is due to the $50 \mathrm{~Hz}$, in the horizontal, vibrations in the 10 to $30 \mathrm{~Hz}$ zone represent a large contribution ; these vibrations correspond to the girder mechanical eigenmodes.
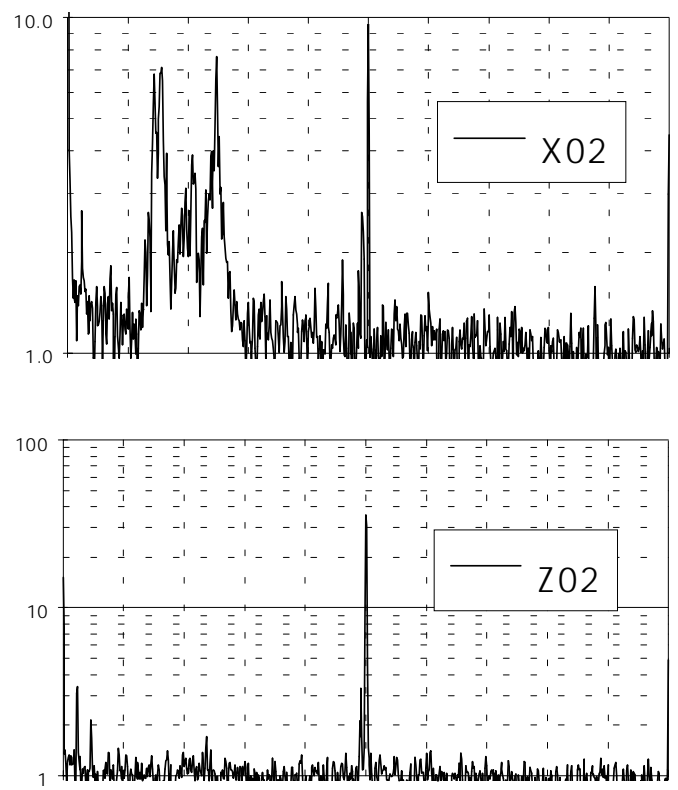

Fig. 3. Typical $\mathrm{X}$ and $\mathrm{Z}$ motion spectrum (vert. scale : $\mu \mathrm{m} \mathrm{rms}$; horiz. scale : 0 to $100 \mathrm{~Hz}$ ).

\subsection{Frequency Response}

The efficiency of the feedback loop has been measured in both planes by comparing the effect of a steering magnet (not included in the loop) with the feedback ON and OFF (see Fig. 4).

In the 2 bunch operating mode, the feedback bandwidth is of about $40 \mathrm{~Hz}$ in the horizontal plane, and of about $150 \mathrm{~Hz}$ in the vertical plane. The difference between the 2 planes is not due to differences in the electronics, but only to the efficiency of the steerers, probably because of the eddy currents in the non isotropic vacuum chamber. Nevertheless, the achieved bandwidth in the horizontal plane ought to be large enough to cure the 10 to $30 \mathrm{~Hz}$ zone vibrations. 

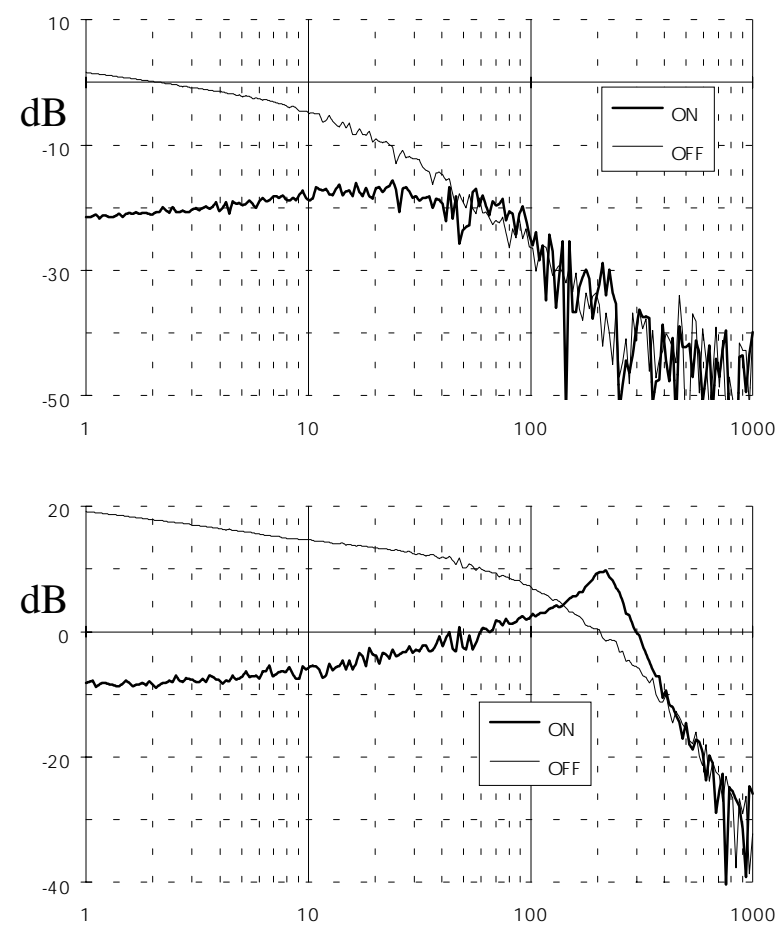

Fig. 4. Feedback bandwidth in the 2 bunch mode. (Upper : horizontal ; lower : vertical).

\subsection{Effects on the Beam Motion}

In the 2 bunch mode, the effect of the feedback is clearly visible on the beam position, both on drifts and on low frequency fluctuations. The later effect is particulary obvious when the beam transport from the linac is powered (in that case, without feedback, one can observe periodic fluctuations at $2.35 \mathrm{~Hz}$ on the beam position, in both planes, which are not explained at the moment).

The effect is also clear on the photon beam monitor installed in the SB3 photon beamline.

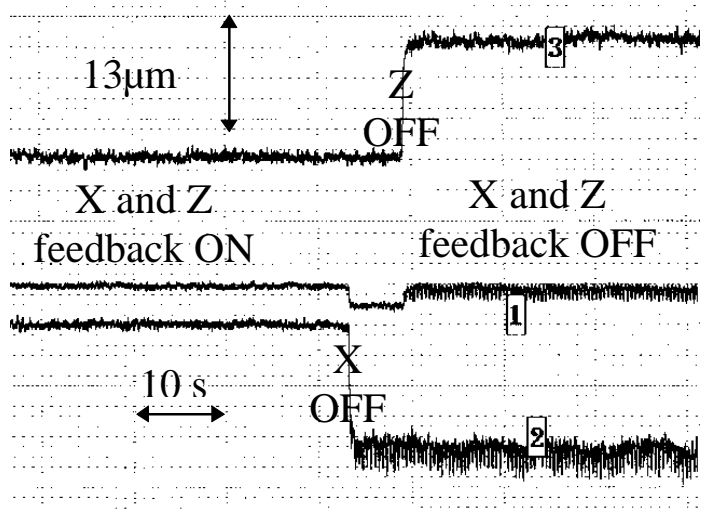

[1] Photon detector SB3 ; [2] BPM X30 ; [3] BPM Z30.

Fig. 5. Position record, feedback ON/OFF.
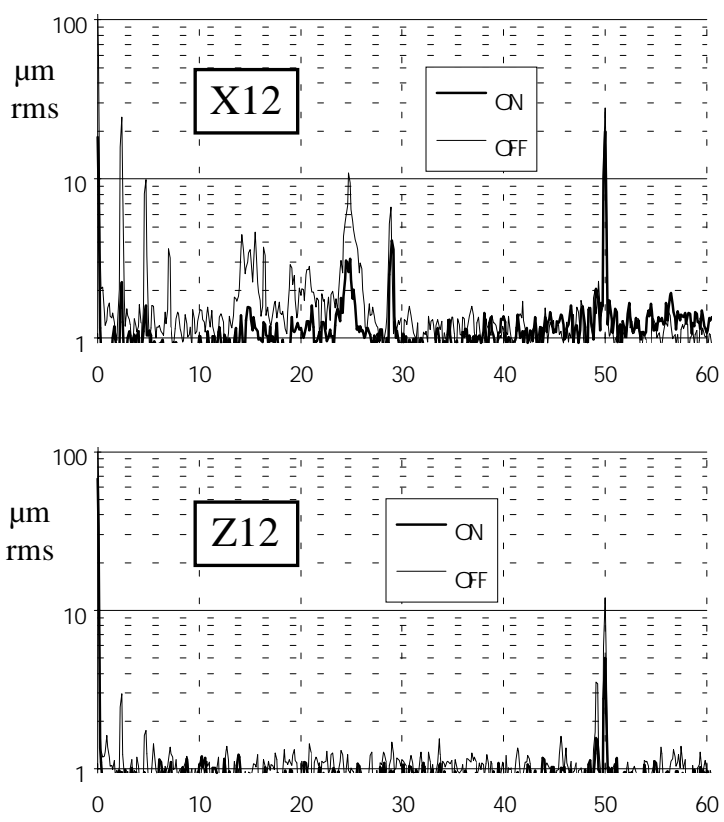

Fig. 6. Spectrum of the beam position signal at station 12 (upper : horizontal ; lower : vertical) feedback ON and OFF.

Most of our tests have been performed in the 2 bunch mode, while only preliminary tests have been carried out in the 24 bunch mode. The first results show that the feedback efficiency is dramatically reduced in the vertical plane with the harmonic matrix usually used in the 2 bunch mode. The SVD matrix seems to be more efficient, but we need to go on with further tests before we are ready to use the feedback for users sessions in the different operating modes.

\section{CONCLUSION}

A fast global beam position feedback system has been implemented and tested on Super-ACO. The achieved bandwidth is $40 \mathrm{~Hz}$ in the horizontal plane and $150 \mathrm{~Hz}$ in the vertical, yielding to a reduction by a factor 10 in the low frquency region. Additional tests are being carried out in order to have the feedback operational during users sessions.

\section{ACKNWOLEDGMENTS}

It is a pleasure to thank all the members of the operation staff of Super-ACO for their collaboration.

\section{REFERENCES}

[1] L. Cassinari, NI/97-08.

[2] K. B. Unser, BIW 96, "New Generation Electronics Applied to Beam Position Monitors".

[3] J.-F. Lamarre, Super-ACO/97-06

[4] J.-F. Lamarre, Super-ACO/98-09. 